\title{
Acoustic and magnetic wave heating in stars
}

\section{The chromospheric emission-magnetic filling factor relation}

\author{
D. Fawzy ${ }^{1}$, K. Stȩpień ${ }^{2}$, P. Ulmschneider ${ }^{1}$, W. Rammacher ${ }^{1}$, and Z. E. Musielak ${ }^{3,1}$ \\ 1 Institut für Theoretische Astrophysik der Universität Heidelberg, Tiergartenstr. 15, 69121 Heidelberg, Germany \\ 2 Warsaw University Observatory, Al. Ujazdowskie 4, 00478 Warszawa, Poland \\ 3 Department of Physics, University of Texas at Arlington, Arlington, TX 76019, USA
}

Received 5 November 2001 / Accepted 14 February 2002

\begin{abstract}
Theoretical chromospheric models described in the two previous papers of this series are used to study the relationship between the chromospheric emission and the filling factor. This theoretically determined relationship shows that the chromospheric emission flux in Ca II $(\mathrm{H}+\mathrm{K})$ is approximately proportional to the square root of the magnetic filling factor at the stellar surface. To relate the filling factor to stellar rotation rate, we compare the theoretical fluxes with observations of stars with known rotation period. The comparison shows that the Rossby number is probably a more appropriate measure of the rotation influence on activity of main-sequence stars than the rotation period. Our theoretical $\mathrm{Mg}$ II $(\mathrm{h}+\mathrm{k})$ and $\mathrm{Ca}$ II $(\mathrm{H}+\mathrm{K})$ emission fluxes are also found to be well correlated, which is in a good agreement with the observational data.
\end{abstract}

Key words. methods: numerical - stars: chromospheres - stars: coronae - stars: magnetic fields - MHD - waves

\section{Introduction}

It has been known for a long time that the chromospheric activity of cool dwarfs is closely correlated with their stellar rotation rate (e.g., Wilson 1968; Noyes et al. 1984; Stẹpień 1989; Strassmeier et al. 1994). Analysis of the $\mathrm{X}$-ray stellar coronal emission has demonstrated that this emission also strongly depends on rotation (e.g., Walter 1982; Stẹpień 1994; Hempelmann et al. 1995). Thus, there is a clear observational evidence that rapidly rotating stars show high levels of chromospheric-coronal activity and that the level of this activity strongly decreases with decreasing stellar rotation rate. The activity-rotation relation is color-dependent (Hartmann et al. 1984; Noyes et al. 1984; Stȩpień 1989, 1993). On the other hand, it is known that the rotation rate of dwarfs depends on their age (Kraft 1967; Skumanich 1972; Baliunas et al. 1995) in a sense that the older the star, the longer is its rotation period. As a result, the activity levels of old, solarlike, stars are considerably lower than those of young stars (Skumanich 1972; Soderblom 1985; Soderblom et al. 1991; Maggio et al. 1987; Güdel et al. 1997).

According to the commonly accepted scenario, the relationship between activity and rotation can be explained by the fact that stellar surface magnetic fields are generated near the bottom of stellar convection zones as a

Send offprint requests to: P. Ulmschneider,

e-mail: ulmschneider@ita.uni-heidelberg.de result of the dynamo mechanism (Parker 1979). Simple considerations predict that the efficiency of dynamo scales with $\tau \Omega$, where $\tau$ is the turnover time in the layers where the dynamo is operating, and $\Omega$ is the angular velocity of these layers (Durney \& Latour 1978; Gray 1982). The turnover time is directly related to the properties of the convection zone, hence it depends on the color of the star. The observations show indeed that the chromospheric and coronal fluxes are tightly correlated with the Rossby number, defined as $R o=P_{\text {rot }} / \tau$, where $P_{\text {rot }}$ is the observed (surface) rotation period. The turnover time can be determined either from theoretical models of stellar convection zone (Gilman 1980; Gilliland 1985; Rucinski \& VandenBerg 1986) or from observations (Noyes et al. 1984; Stępień 1989, 1994; Saar 1998).

The generated magnetic fields, which emerge at stellar photospheres, interact with turbulent motions in the uppermost layers of stellar convection zones giving rise to magnetic activity. In particular, the strength of the fields and their geometry directly influence the heating rates of chromospheres and coronae. Hot coronae evaporate into space and the resulting stellar winds carry with them the frozen-in magnetic fields. The winds also carry away angular moment which brakes the stellar rotation. Stars are born as fast rotators (Soderblom et al. 1991; Stauffer 1994) with a very high level of activity and intense angular momentum loss rate. But when stars age and spin down, the efficiency of their dynamos diminishes and this results in 
a decrease of the magnetic activity. Therefore, old stars have long rotation periods and minimum activity levels.

While this general scenario appears to be correct, many details are still poorly known and need a thorough investigation. One of these involves the process of chromospheric heating and its dependence on the amount of the magnetic flux present at the photospheric level. In the first two papers of this series (Fawzy et al. 2002a,b, henceforth called Papers I, II; see also Ulmschneider et al. 2001a), we have calculated theoretical chromospheric models and used these models to compute the emerging radiative fluxes. Our approach is based solely on first principles and only the basic stellar parameters, namely, effective temperature $T_{\text {eff }}$, gravity $g$ and chemical composition $Z_{\mathrm{M}}$ (solar abundances), are specified. The only free parameter in these calculations is the filling factor $f$ describing the fraction of the stellar surface covered by magnetic fields.

The results presented in Papers I and II show that the calculated $\mathrm{Ca}$ II $\mathrm{H}+\mathrm{K}$ emission fluxes agree well with the observations of stars with spectral types ranging from $\mathrm{F}$ to $\mathrm{M}$, both in absolute values, in the $T_{\text {eff }}$-dependence, and in the range of variation. In case of the $\mathrm{Mg}$ II $\mathrm{h}+\mathrm{k}$ emission, the absolute values of the theoretical fluxes agree well with the observational data, including the $T_{\text {eff-dependence and }}$ their lower limit, which corresponds in our models to pure acoustic heating. Yet the upper limit obtained from our $\mathrm{Mg}$ II calculations is persistently lower than the observations. This is a clear indication that additional heating processes, not included in our wave models (for example, the direct dissipation of magnetic fields in the most active stars) take place in the high chromosphere, where Mg II lines are formed.

In this paper, we used our theoretical chromosphere models to determine the dependence of the theoretical $\mathrm{Ca}$ II and Mg II emission fluxes on the filling factor. In an attempt to relate the filling factor to the rotation rate, we also compare the theoretical fluxes with observations of stars with known rotation period. Finally, the theoretical flux-flux relation is compared to the one established observationally and the resulting differences are discussed. Our theoretical approach is briefly described in Sect. 2 while Sect. 3 is devoted to the observational data. Our main results are presented and discussed in Sect. 4 and conclusions are given in Sect. 5 .

\section{Theoretical chromosphere models}

Our theoretical, time-dependent and two-component chromospheric models take into account non-magnetic regions (heated by acoustic waves) and magnetic flux tubes (assumed uniformly distributed and heated by longitudinal tube waves). The coverage of the stellar surface by magnetic fields, specified by the surface area filling factor $f$ is a free parameter in these calculations. The initial wave energy fluxes are input parameters for the models and they are calculated by using the LighthillStein theory (Lighthill 1952; Stein 1967) for the generation of acoustic waves in stellar convection zones
(Musielak et al. 1994; Ulmschneider et al. 1996) and a numerical method (Ulmschneider \& Musielak 1998) for the generation magnetic waves in stellar atmospheres (Ulmschneider et al. 2001b). To account for the energy carried by transverse tube waves (Huang et al. 1995; Musielak \& Ulmschneider 2001, 2002), the longitudinal wave fluxes are increased by a factor $M$, where for a realistic contribution we take $M=5$. The acoustic, $F_{\mathrm{A}}$, and longitudinal, $F_{\mathrm{L}}$, wave energy fluxes and the wave periods $P_{\mathrm{A}}$ and $P_{\mathrm{L}}$, corresponding to the maximum wave energy fluxes carried by these waves, used in our calculations are given in Tables 1 and 2 of Paper I.

A time-dependent magneto-hydrodynamic code based on the method of characteristics is used to compute the propagation of both acoustic waves in a plane-parallel atmosphere (e.g. Ulmschneider et al. 1977; Buchholz et al. 1998) and longitudinal waves along the magnetic flux tubes (e.g. Herbold et al. 1985). In these wave computations we consider radiative losses (see Paper I) by the $\mathrm{H}^{-}$continuum and the $\mathrm{Mg}$ II, Ca II and Fe II emission lines and solve the appropriate radiative transfer equations together with the statistical equilibrium equations for NLTE populations (Ulmschneider 1994; Hünerth \& Ulmschneider 1995).

The time-dependent energy balance between the wave dissipation by shocks and the emitted radiative losses determines the local values of temperature, density and pressure in the magnetic and non-magnetic stellar atmosphere regions. After the wave computation is completed the intensities and radiation fluxes emerging from the stars covered by a flux tube forest are computed using a multiray radiative transfer code. It must be noted that earlier versions of these models have been used by Buchholz et al. (1998) to predict theoretically the level of the "basal flux" and by Cuntz et al. (1999) to study the decrease of chromospheric activity with stellar rotation for K2 V stars.

A series of theoretical chromospheric models has been calculated for late-type dwarfs with the magnetic filling factor ranging from $f=0.0$, for purely acoustically heated chromospheres, to $f=0.4$, for magnetically heated chromospheres which outside the flux tubes are heated by acoustic waves. As discussed in Paper II, the pure acoustically heated chromospheres give the emerging Ca II emission fluxes that agree reasonably well with the "basal flux" level of chromospheric activity. However, the models with the heating by both magnetic and acoustic waves (with $f=0.4$ and $M=5$ ) well describe the upper limit of the chromospheric activity. Figure 8 of Paper II shows the behavior of the total $F_{\mathrm{H}+\mathrm{K}}$ flux as function of color and filling factor (see also Table 1 below). Our results presented in Papers I and II give us confidence that our theoretical models describe correctly the chromospheres of solartype stars with low and moderate levels of stellar activity. Therefore, we use these models in this paper to study the relationship between the chromospheric emission and the magnetic filling factor. 


\section{Observational data}

\subsection{Observed magnetic filling factors}

It would be most natural to compare our theoretical filling factors directly with the observed stellar magnetic fields. The observations give the strength of the magnetic field prevailing on the stellar surface $B_{\text {obs }}$ and the filling factor $f_{\text {obs }}$ (Marcy 1984; Saar 1988). Their product gives the average surface intensity of the magnetic field, sometimes also called the unsigned magnetic surface flux. Early observations suggested that $B_{\mathrm{obs}}$ is equal to the equipartition field $B_{\text {eq }}$ (the field with the magnetic pressure equal to the gas pressure at the photospheric level) hence it should depend only on the color of a star. This ought to leave $f_{\text {obs }}$ as the only parameter depending on rotation. In spite of the significant observational scatter, the results showed that $f_{\text {obs }}$ correlates indeed very well with rotation (Stȩpien 1991; Saar 1991; Montesinos \& Jordan 1993; Jordan 1997). The best fits were obtained assuming the exponential dependence of $f_{\text {obs }}$ on the Rossby number

$\log f_{\mathrm{obs}}=a+b R o$,

with $a \approx-0.06$ and $b \approx 0.8$ (Stȩpień 1991; Montesinos \& Jordan 1993) but a nearly equally good correlation could be obtained between $B_{\text {obs }} f_{\text {obs }}$ and $R o$ (Montesinos \& Jordan 1993; Saar 1991). Later measurements showed that $B_{\text {obs }}$ depends weakly on the rotation rate for the most active stars (Saar 1996) and in some cases even two different values of $B_{\text {obs }}$ were obtained for one star, corresponding probably to a typical spot field and a typical plage field (Saar 1992). It is also well known that the newer observational techniques did not get fully consistent results with the older ones (Valenti et al. 1995; Rüedi et al. 1997). In addition, for several late type stars with measured surface magnetic fields the chromospheric emission fluxes are not known. All this severely restricts the direct comparison of the observed and theoretical filling factors.

Cuntz et al. (1998) used values of the surface magnetic field for several stars with known rotation periods, obtained by Rüedi et al. (1997), to determine an empirical period-magnetic field relation which then was used to relate the theoretical chromospheric flux to rotation rate (Cuntz et al. 1999). They derived a linear relation between $B_{\text {obs }} f_{\text {obs }}$ and $P_{\text {rot }}$ of the form

$B_{\text {obs }} f_{\text {obs }}=238-5.51 P_{\text {rot }}$,

for stars with $10 \leq P_{\text {rot }} \leq 40$ days. Unfortunately, it is impossible to derive period-magnetic field relations for individual spectral types due to a low number of available data.

There is, however, a relatively large number of stars for which rotation periods and chromospheric fluxes have been determined. A comparison of the observed emission fluxes of these stars with the theoretical values will permit us to link the rotation period to the filling factor $f$.

We searched the literature for single dwarfs with known rotation periods and measured chromospheric flux

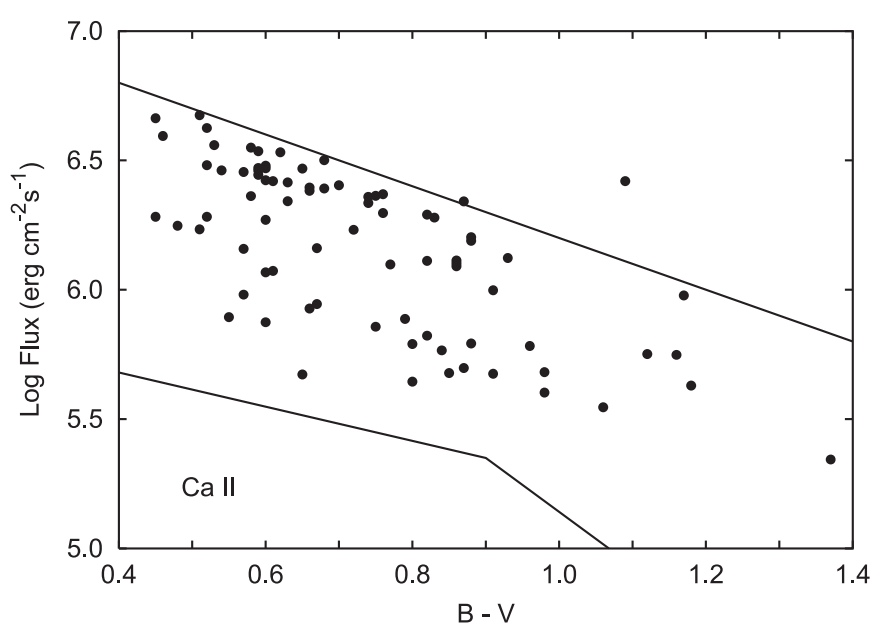

Fig. 1. The distribution of stellar Ca II emission fluxes with color for single dwarfs with known rotation periods. The upper and lower limit for stellar chromospheric flux, as determined in Paper II, are shown as solid lines.

in Ca II and/or Mg II line cores. We have found about 80 stars with measured calcium emission flux and about 40 with magnesium emission fluxes. Our main sources of data were the papers by Stȩpień (1994), Saar \& Brandenburg (1999) and Messina et al. (2001), with a couple of periods taken from Stȩpień \& Geyer (1996). References to original determinations of rotation periods and chromospheric fluxes can be found in the first three papers.

\section{2. $\mathrm{Ca} / / \mathrm{H}+\mathrm{K}$ emission fluxes}

Ca II emission fluxes of stars with known rotation periods represent our primary set of observational data for comparison with those obtained from our theoretical models. Figure 1 shows the emission fluxes of the selected stars and their comparison to the upper and lower observational limits of the calcium emission as determined in Paper II. It is seen that these stars have a wide range of fluxes, representing different levels of the chromospheric activity. Unfortunately, they are not distributed evenly in color; a great majority of them have $B-V<0.9$. Now, the theoretical results presented in Paper II have been obtained for stars with 6 different effective temperatures (see Table 1 in Paper II). Because of the lack of the observational data for the highest and the lowest temperature, corresponding to $B-V=0.44$ and 1.40 , respectively, we cannot relate the observations to the theoretical fluxes. Fortunately, there exist enough observational data for the other effective temperatures. Table 1 contains the coefficients of the linear fits of the type $\log F_{\mathrm{HK}}=a+b P_{\text {rot }}$, where $F_{\mathrm{HK}}$ is the observed net calcium emission flux (see Paper II for details). The coefficients will be used to infer the relation between the rotation period and the filling factor used in theoretical computations.

The stars with known rotation periods and $\mathrm{Mg}$ II fluxes are distributed similarly in color (in fact, with a few exceptions they are a subset of the previous set). 
Table 1. Coefficients of the linear fits of the Ca II H+K emission flux $\log F_{\mathrm{HK}}=a+b P_{\text {rot }}$ for $B-V$ values corresponding to the model calculations.

\begin{tabular}{cccc}
\hline \hline$\langle B-V\rangle$ & $N$ & $a$ & $b$ \\
\hline 0.58 & 20 & $6.617 \pm 0.052$ & $-0.0343 \pm 0.0051$ \\
0.68 & 15 & $6.603 \pm 0.038$ & $-0.0258 \pm 0.0024$ \\
0.81 & 15 & $6.466 \pm 0.028$ & $-0.0178 \pm 0.0010$ \\
1.15 & 6 & $6.290 \pm 0.122$ & $-0.0179 \pm 0.0042$ \\
\hline
\end{tabular}

Table 2. Coefficients of the linear fits of the $M g I I h+k$ emission flux $\log F_{\mathrm{hk}}=a+b P_{\text {rot }}$ for $B-V$ values corresponding to the model calculations.

\begin{tabular}{cccc}
\hline \hline$\langle B-V\rangle$ & $N$ & $a$ & $b$ \\
\hline 0.58 & 11 & $6.734 \pm 0.067$ & $-0.0363 \pm 0.0057$ \\
0.68 & 5 & $6.610 \pm 0.071$ & $-0.0163 \pm 0.0046$ \\
0.81 & 9 & $6.682 \pm 0.072$ & $-0.0200 \pm 0.0034$ \\
1.15 & 4 & $6.474 \pm 0.118$ & $-0.0208 \pm 0.0047$ \\
\hline
\end{tabular}

The coefficients are given in Table 2. Because of the lower number of data standard errors are here larger than in Table 1.

\section{Results}

\subsection{Ca II Emission flux versus magnetic filling factor}

The results of our calculations show that the magnetic filling factor strongly affects the level of $\mathrm{Mg}$ II $\mathrm{h}+\mathrm{k}$ and $\mathrm{Ca}$ II $\mathrm{H}+\mathrm{K}$ emission in the sense that with the increasing $f$ the emission flux also increases (Paper II). Tables 3 and 4 give the relevant theoretical data for the calcium emission fluxes taken from Paper II. The Ca II data are also displayed in Fig. 2. Linear fits of the form $\log F=a+b \log f$ could be made to these theoretical fluxes and their coefficients are given in Table 5 .

The errors listed here refer to the internal scatter among the theoretical models. They do not include deviations of our (highly idealized) models from the actual conditions existing in real stars. We expect such external uncertainties to be significantly larger.

Table 3. Theoretical total $\mathrm{Ca}$ II $\mathrm{H}$ and $\mathrm{K}$ emission fluxes $\log F_{\mathrm{H}+\mathrm{K}}$ for stars of different spectral type, effective temperature $T_{\text {eff }}$, color $B-V$ and magnetic filling factors $f$ with $M=5$.

\begin{tabular}{lccccccc}
\hline \hline SpT & $T_{\text {eff }}$ & $B-V$ & .00 & .05 & .10 & .20 & .40 \\
\hline F5V & 6440 & 0.44 & 6.01 & 6.21 & 6.31 & 6.44 & 6.52 \\
G0V & 6030 & 0.58 & 5.95 & 6.04 & 6.17 & 6.26 & 6.43 \\
G5V & 5770 & 0.68 & 5.83 & 5.95 & 6.02 & 6.14 & 6.27 \\
K0V & 5250 & 0.81 & 5.66 & 5.77 & 5.83 & 5.92 & 6.11 \\
K5V & 4350 & 1.15 & 5.02 & 5.38 & 5.65 & 5.72 & 5.86 \\
M0V & 3850 & 1.40 & 4.13 & 4.50 & 4.64 & 5.24 & 5.56 \\
\hline
\end{tabular}

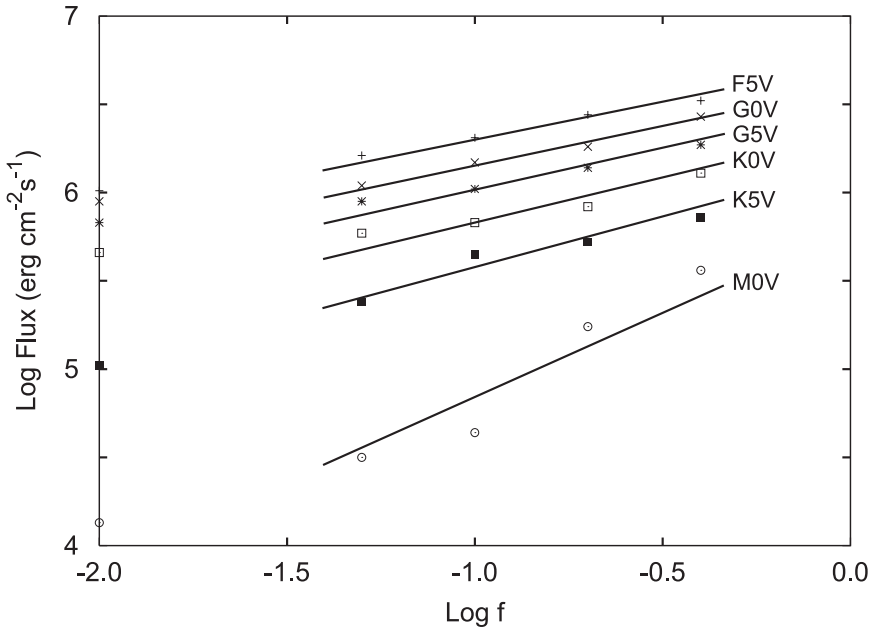

Fig. 2. Linear fits of the theoretical emitted fluxes $\log F_{\mathrm{H}+\mathrm{K}}$ in Ca II versus the filling factor $\log f$ for magnetic wave heated late-type stars with $M=5$. Also displayed are the fluxes for acoustical wave heated stars by arbitrarily placing the data at $\log f=-2.0$.

Table 4. Theoretical total $\mathrm{Mg}$ II $\mathrm{h}$ and $\mathrm{k}$ emission fluxes $\log F_{\mathrm{h}+\mathrm{k}}$ for stars of different spectral type, effective temperature $T_{\text {eff }}$, color $B-V$ and magnetic filling factors $f$ with $M=5$.

\begin{tabular}{lcccc}
\hline \hline SpT & $T_{\text {eff }}$ & $B-V$ & .00 & .40 \\
\hline F5V & 6440 & 0.44 & 5.94 & 6.44 \\
G0V & 6030 & 0.58 & 5.81 & 6.36 \\
G5V & 5770 & 0.68 & 5.86 & 6.21 \\
K0V & 5250 & 0.81 & 5.63 & 6.15 \\
K5V & 4350 & 1.15 & 5.33 & 6.02 \\
M0V & 3850 & 1.40 & 4.86 & 5.85 \\
\hline
\end{tabular}

Table 5. Coefficients of the linear fits $\log F_{\mathrm{H}+\mathrm{K}}=a+b \log f$, for late-type stars, with $M=5$.

\begin{tabular}{lccc}
\hline \hline Star & $T_{\text {eff }}$ & $a$ & $b$ \\
\hline F5V & 6440 & $6.669 \pm 0.020$ & $0.352 \pm 0.022$ \\
G0V & 6030 & $6.581 \pm 0.032$ & $0.419 \pm 0.035$ \\
G5V & 5770 & $6.400 \pm 0.030$ & $0.359 \pm 0.033$ \\
K0V & 5250 & $6.221 \pm 0.064$ & $0.369 \pm 0.070$ \\
K5V & 4350 & $6.079 \pm 0.085$ & $0.502 \pm 0.093$ \\
M0V & 3850 & $6.052 \pm 0.181$ & $1.256 \pm 0.198$ \\
\hline
\end{tabular}

\subsection{Rotation-filling factor relation}

Based on the computed $\mathrm{Ca}$ II $\mathrm{H}+\mathrm{K}$ and $\mathrm{Mg}$ II $\mathrm{h}+\mathrm{k}$ fluxes for stars of different magnetic activities and an observed empirical relation between the emitted flux and rotation period, we estimate the filling factors of stars with different rotation periods. Because the observational data for $\mathrm{Ca}$ II $\mathrm{H}+\mathrm{K}$ fluxes are more numerous, we expect to obtain more accurate results from their comparison with theoretical fluxes. The coefficients of the linear fits of the type $\log f=a+b P_{\text {rot }}$, resulting from this comparison of calcium emission flux for four different spectral types, are 
Table 6. Coefficients of the theoretical linear fits $\log f=a+$ $b P_{\text {rot }}$ and $\log B f=c+b P_{\text {rot }}$, for late-type stars of given color index $B-V$ for magnetic wave fluxes with $M=5$. Also shown is the magnetic field strength $B(\mathrm{G})$ at the stellar surface of the flux tube models employed (see Paper I).

\begin{tabular}{lccccc}
\hline \hline Star & $B-V$ & $B(G)$ & $a$ & $b$ & $c$ \\
\hline G0V & 0.58 & 1570 & 0.087 & -0.082 & 3.283 \\
G5V & 0.68 & 1740 & 0.568 & -0.072 & 3.809 \\
K0V & 0.81 & 1850 & 0.665 & -0.048 & 3.932 \\
K5V & 1.15 & 2370 & 0.422 & -0.035 & 3.797 \\
\hline
\end{tabular}

given in Table 6 . The magnesium data give similar fits but the present data are significantly less numerous and less accurate, and because the variation of $f$ does not represent the full spread of the chromospheric emission variability, we do not list the coefficients.

As it is seen from Fig. 2 and Table 5, the calcium emission flux is proportional to $f^{b}$, where $b \approx 0.4$ for all the investigated spectral types except M0V. The solar observations indicate that $b \approx 0.6 \pm 0.14$ (Schrijver et al. 1989). The stellar data give a similar value (Stȩpień 1994). Taking into account all the uncertainties (and the fact that the formally calculated average value of the exponent for all the modeled stellar atmospheres, including the M0V star, is equal to 0.54), we conclude that the theoretical results are in a fair agreement with the observational data.

The linear fits given in Table 6 relate the (theoretical) filling factors and magnetic fluxes to the rotation period. Because the theoretical results for magnetic wave fluxes with $M=5$ used in the present paper give calcium emission fluxes slightly lower than the observations (see Paper II), the terms $a$ of the linear relations between $\log f$ and $P_{\text {rot }}$, listed in Table 6 , are probably too large. Only in case of $B-V=0.44$ is this term close to zero, as expected. Introducing the $B$ values from Paper I into the relation $\log f=a+b P_{\text {rot }}$, we find that the resulting relation $\log B f=c+b P_{\text {rot }}$ is in fair agreement with the observationally established relationship given by Eq. (2), particularly if one takes into account the completely different approach adopted in deriving both formulae and the large scatter of the observational data. The coefficients $b$ decrease with advancing spectral type which indicates that the filling factor decreases faster with the rotation period in hotter than in cooler stars. This reflects the fact that the Rossby number is a more appropriate measure of the rotation influence on activity of main sequence stars than the rotation period. In fact, one can determine for each spectral type purely empirical turnover times $\tau \sim 1 / b$ and dividing $P_{\text {rot }}$ by the corresponding $\tau$ one obtains a single relation between $f$, or $B f$ of stars of all considered spectral types and the thus defined Rossby number. These empirical turnover times agree with other determinations (see Introduction) but, in our opinion, are less accurate.

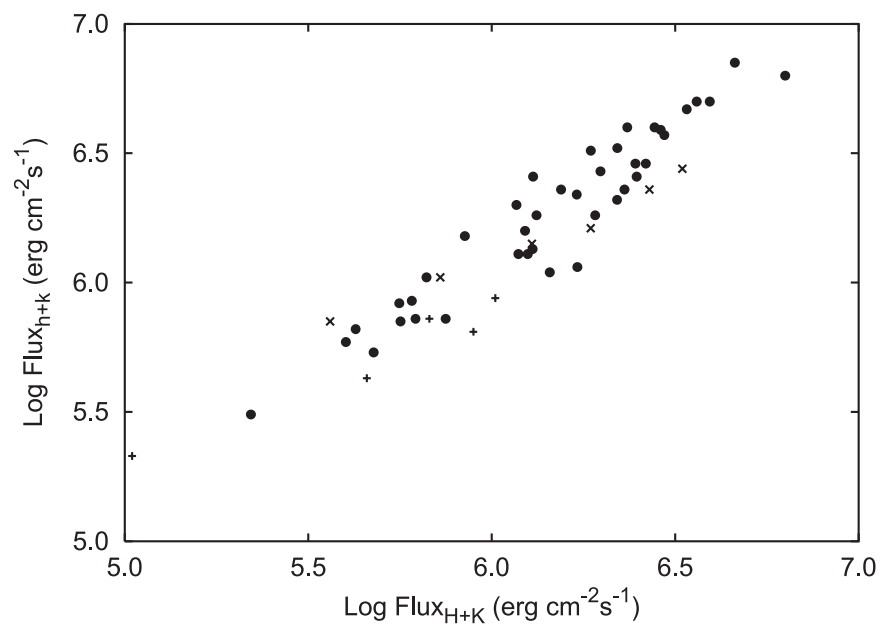

Fig. 3. The relation between the observed $\mathrm{Ca}$ II $\mathrm{H}+\mathrm{K}$ and $\mathrm{Mg}$ II $\mathrm{h}+\mathrm{k}$ fluxes of the periodic stars (dots). Also shown are the theoretical relations for stars with pure acoustic heating (indicated by + ) and with magnetic heating $(f=0.4, M=5$, indicated by $\times$ ). The theoretical fluxes are from Tables 3 and 4 .

\subsection{Relation between $\mathrm{Mg} / /$ and Ca I/ emission fluxes}

The radiative emission fluxes originating from the different outer atmospheric layers are found to be highly correlated for the majority of cool stars as shown by many authors (e.g. Ayres et al. 1981; Oranje 1985; Rutten et al. 1991). In all investigated cases power relations can be fitted to flux-flux relations. In case of $\mathrm{Ca}$ II $\mathrm{H}+\mathrm{K}$ and $\mathrm{Mg}$ II $\mathrm{h}+\mathrm{k}$ fluxes the observations indicate that the power should be close to 1 (Hartmann et al. 1984; Rutten et al. 1991). We plotted in Fig. 3 the logarithm of both fluxes for our stars. A linear fit gives

$\log F_{\mathrm{hk}}=(0.95 \pm 0.05) \log F_{\mathrm{HK}}+(0.40 \pm 0.30)$.

We conclude from Fig. 3 that the fluxes are strictly proportional to one another within the accuracy of the fit.

The relation between the theoretical $\mathrm{Mg}$ II and Ca II emission fluxes for main sequence stars taken from Tables 3 and 4 is also displayed in Fig. 3 for different levels of magnetic activity. Here we have chosen only the cases of pure acoustic wave heating $(f=0.0$, indicated by + ) and of magnetic wave heating $(f=0.4, M=5$, indicated by $\times)$. It is seen that there appears to be no major discrepancy with the observed flux-flux relation.

That there is a flux-flux relation is not surprising, because in our wave heated chromosphere models both radiative emissions emerge from the same hot post-shock regions of the dissipating waves. However, because the $\mathrm{Mg}$ II and Ca II emissions have different dependences on the temperature (e.g. Kalkofen et al. 1984), with the Mg II losses more strongly concentrated to the regions of highest temperature, the deviation from the proportional flux-flux relation could give us important information about the shape of the waves. Such detailed studies must be deferred to future investigations. Nevertheless, it is rewarding to see that taking monochromatic waves for our present 
exploratory calculations appears to give reasonable overall agreement with the observations.

\section{Conclusions}

We have compared observed chromospheric emission fluxes in the $\mathrm{Ca}$ II $\mathrm{H}+\mathrm{K}$ and $\mathrm{Mg}$ II $\mathrm{h}+\mathrm{k}$ lines of late-type main sequence stars with theoretical simulations. The theoretical calculations were based completely on first principles, that is, we only specify the four independent stellar parameters: effective temperature $T_{\text {eff }}$, gravity $g$, metal abundance $Z_{\mathrm{M}}$ (we took solar-like population I abundances), and magnetic filling factor $f$ at the stellar surface. With these four parameters, which uniquely identify a star, we constructed convection zone models and models of the magnetic flux tube forest which homogeneously covers the stellar surface. Here we assumed that the magnetic field occurs mainly in the form of thin flux tubes, which at the stellar surface have diameters equal to the local scale height. The magnetic field strength at this height is assumed to be given by a fixed fraction of the equipartition field strength, which is determined by the external gas pressure.

Theoretical wave energy spectra and fluxes of acoustic and longitudinal tube waves as well as wave periods have been computed. To take into account the amount of energy carried by transverse waves, we increased the longitudinal wave fluxes by a factor $M$, where for a realistic contribution we take $M=5$. These waves were allowed to propagate in the two-component outer atmosphere model and dissipate their energy by forming shock waves. With a multi-ray transfer code, we have simulated line profiles of the $\mathrm{Ca} \mathrm{II} \mathrm{H}+\mathrm{K}$ and $\mathrm{Mg}$ II $\mathrm{h}+\mathrm{k}$ lines emerging from the two-dimensional tube forest and have compared them with observations. A number of interesting conclusions resulting from this comparison have been given in Papers I and II.

In this paper, we used our theoretical chromosphere models to determine the dependence of the theoretical Ca II and Mg II emission fluxes on the filling factor. In an attempt to relate the filling factor to the rotation rate, we also compare the theoretical fluxes with observations of stars with known rotation period. In addition, the theoretical flux-flux relation is compared to the one established observationally. The obtained results lead to the following conclusions:

1. The theoretical relation between the magnetic filling factor and the chromospheric emission flux predicts that the emission flux is approximately proportional to the square root of the filling factor. The predicted relation is in a fair agreement with observations.

2. Using the observations of stars with known emission flux and the rotation period it was possible to relate the theoretical filling factor and the theoretical magnetic flux to the rotation period. The relations are color dependent: the slope of the filling factor-rotation period decreases from hotter to cooler stars suggesting that the Rossby number is probably a more appropriate measure of the rotation influence on activity of main-sequence stars than the rotation period.

3. The observations of chromospherically active stars with known rotation periods show that the $\mathrm{Mg}$ II and Ca II emission fluxes are strictly proportional to one another. The theoretical results are in a good agreement with the observational data.

Acknowledgements. This work was supported by NSF under grant ATM-0087184 (Z.E.M. and P.U.), by the KBN grant 5 P03D 00621 (K.S.), by the DFG grants Ul57/25-3, Ul57/301 and by NATO under grant CRG-910058 (P.U. and Z.E.M.). Z.E.M. also acknowledges the support of this work by the Alexander von Humboldt Foundation.

\section{References}

Ayres, T. R., Marstad, N. C., \& Linsky, J. L. 1981, ApJ, 247, 545

Baliunas, S. L., Donahue, R. A., Soon, W. H., et al. 1995, ApJ, 438, 269

Buchholz, B., Ulmschneider, P., \& Cuntz, M. 1998, ApJ, 494, 700

Cuntz, M., Ulmschneider, P., \& Musielak, Z. E. 1998, ApJ, 493, L117

Cuntz, M., Rammacher, W., Ulmschneider, P., Musielak, Z. E., \& Saar, S. H. 1999, ApJ, 522, 1053

Durney, B. R., \& Latour, J. 1978, Geophys. Astrophys. Fluid Dyn., 9, 241

Fawzy, D., Rammacher, W., Ulmschneider, P., Musielak, Z. E., \& Stępień K. 2002a, A\&A, 386, 971 (Paper I)

Fawzy, D., Ulmschneider, P., Stȩpień, K., Musielak, Z. E., \& Rammacher, W. 2002b, A\&A, 386, 983 (Paper II)

Gray, D. F. 1982, ApJ, 261, 259

Gilliland, R. L. 1985, ApJ, 299, 286

Gilman, P. 1980, in Stellar Turbulence, ed. D. F. Gray, \& J. L. Linsky (Springer, Berlin), 19

Güdel, M., Guinan, E. F., \& Skinner, S. L. 1997, ApJ, 483, 947

Hartmann, L. W., Baliunas, S. L., Duncan, D. K., \& Noyes, R. W. 1984, ApJ, 279, 778

Hempelmann, A., Schmitt, J. H. M. M., Schultz, M., Rüdiger G., \& Stępień, K. 1995, A\&A, 294, 515

Herbold, G., Ulmschneider, P., Spruit, H. C., \& Rosner, R. 1985, A\&A, 145, 157

Hünerth, G., \& Ulmschneider, P. 1995, A\&A, 293, 161

Jordan, C. 1997, Astron. Geophys., 38, 10

Kalkofen, W., Ulmschneider, P., \& Schmitz, F. 1984, ApJ, 287, 952

Kraft, R. P. 1967, ApJ, 150, 551

Lighthill, M. J. 1952, Proc. Roy. Soc. London A, 211, 564

Maggio, A., Sciortino, S., Vaiana, G. S., et al. 1987, ApJ, 315, 687

Marcy, G. W. 1984, ApJ, 276, 286

Messina, S., Rodonó, M., \& Guinan, E. F. 2001, A\&A, 366, 215

Montesinos, B., \& Jordan, C. 1993, MNRAS, 264, 900

Musielak, Z. E., Rosner, R., Stein, R. F., \& Ulmschneider, P. 1994, ApJ, 423, 474

Musielak, Z. E., \& Ulmschneider, P. 2001, A\&A, 370, 541

Musielak, Z. E., \& Ulmschneider, P. 2002, A\&A, 386, 606 
Noyes, R. W., Hartmann, L. W., Baliunas, S. L., Duncan, D. K., \& Vaughan, A. H. 1984, ApJ, 279, 763

Oranje, B. J. 1985, A\&A, 154, 185

Parker, E. N. 1979, Cosmical Magnetic Fields (Oxford University Press)

Rucinski, S. M., \& VandenBerg, D. A. 1986, PASP, 98, 669

Rüedi, I. Solanki, S. K., Mathys, G., \& Saar, S. H. 1997, A\&A, 318,429

Rutten, R. G. M., Schrijver, C. J., Lemmens, A. F. P., \& Zwaan, C. 1991, A\&A, 252, 203

Saar, S. H. 1988, ApJ, 324, 441

Saar, S. H. 1991, in The Sun and Cool Stars: activity, magnetism, dynamos, ed. I. Tuominen, D. Moos, \& G. Rüdiger (Springer, Berlin), 389

Saar, S. H. 1992, in Cool Stars, Stellar Systems, and the Sun, ASP Conf. Ser. 26, ed. M. S. Giampapa, \& J. A. Bookbinder, 252

Saar, S. H. 1996, in Magnetodynamic Phenomena in the Solar Atmosphere-Prototypes of Stellar Magnetic Activity, ed. Y. Uchida, T. Kosugi, \& H. S. Hudson (Kluwer, Dordrecht), 367

Saar, S. H. 1998, in Cool Stars, Stellar Systems, and the Sun, ed. R. A. Donahue, \& J. A. Bookbinder, ASP Conf. Ser., 154,211

Saar, S. H., \& Brandenburg, A. 1999, ApJ, 524, 295

Schrijver, C. J., Cote, J., Zwaan, C., \& Saar, S. H. 1989, ApJ, 337,964

Skumanich, A. 1972, ApJ, 171, 565
Soderblom, D. R. 1985, AJ, 90, 2103

Soderblom, D. R., Duncan, D. K., \& Johnson, D. R. H. 1991, ApJ, 375, 722

Stauffer, J. R. 1994, in Cool Stars, Stellar Systems, and the Sun, ASP Conf. Ser. 64, ed. J.-P. Caillault, 163

Stein, R. F. 1967, Solar Phys., 2, 385

Stȩpień, K. 1989, A\&A, 210, 273

Stȩpień, K. 1991, Acta Astr., 41, 1

Stępień, K. 1993, in The Cosmic Dynamo, ed. F. Krause, K. H. Badler, \& G. Rudiger (Kluwer, Dordrecht), 141

Stępień, K. 1994, A\&A, 292, 191

Stẹpień, K. \& Geyer E. 1996, A\&AS, 117, 83

Strassmeier, K. G., Handler, G., Paunzen, E., \& Rauth, M. 1994, A\&A, 281, 855

Ulmschneider, P. 1994, A\&A, 288, 1021

Ulmschneider, P., Fawzy, D. E., Musielak, Z. E., \& Stẹpień, K. 2001a, ApJ, 559, L167

Ulmschneider, P., Kalkofen, W., Nowak, T., \& Bohn, U. 1977, A\&A, 54, 61

Ulmschneider, P., \& Musielak, Z. E. 1998, A\&A, 338, 311

Ulmschneider, P., Musielak, Z. E., \& Fawzy, D. E. 2001b, A\&A, 374,662

Ulmschneider, P., Theurer, J., \& Musielak, Z. E. 1996, A\&A, 315,212

Valenti, J. A., Marcy, G.W., \& Basri, G. 1995, ApJ, 439, 939

Walter, F. M. 1982, ApJ, 253, 745

Wilson, O. C. 1968, ApJ, 153, 221 